\title{
Amalgam and resin composite use in the UK
}

\author{
Amalgam and composite use in UK general dental practice in 2001 \\ F. J. T. Burke, S. McHugh, A. C. Hall, R. C. Randall, E. Widstrom and H. Forss Br Dent J 2003; 194: 613-618
}

Objective

This study determined the reasons for dentists' choice of materials, in particular amalgam and resin composite, in Great Britain.

\section{Method}

A questionnaire was developed to elicit this information. The names and addresses of 1,000 UK-based dentists were selected at random. The questionnaire was mailed to these dentists with an explanatory letter and reply-paid envelope.

\section{Results}

Six hundred and fifty four replies were received. Regarding choice of material, 100\% of respondents cited clinical indication as the most influencial factor, although patients' aesthetic demands (99\%), patients' choice (95\%) and patients' financial situation (92\%) were also reported to influence respondents' choice. Thirty-five per cent of respondents used composite 'sometimes', 15\% 'often', and 1\% 'always' in extensive loadbearing cavities in molar teeth. For composite restorations in posterior teeth, 92\% 'always', 'often' or 'sometimes' used the total etch technique and 53\% never used rubber dam. Seventy per cent of respondents agreed with the statement 'discontinuation of amalgam restricts a dentist's ability to adequately treat patients'. Eighty-one per cent considered that the growth in the use of composites increased the total cost of oral healthcare.

\section{Conclusions}

Forty nine per cent of the respondents from England and Wales seldom or never place large composite restorations in molar teeth. Their choice of material is influenced greatly by clinical indications, and patients' aesthetic demands.

\section{IN BRIEF}

- The use of resin composite materials had increased in the five years preceding the survey in $62 \%$ of practices.

- Regarding choice of materials, clinical indications was an important factor, but patients' aesthetic demands were also highly relevant.

- Half of the respondents place large composite restorations in posterior teeth.

\section{COMMENT}

This survey assessed practitioners' perceptions and use of amalgam and composites in the restoration of posterior teeth. In common with other studies of this type, which present findings based on the responses of hundreds of practitioners, this paper provides valuable insight into the materials and techniques used in everyday clinical practice. Despite the use of composites having increased in $62 \%$ of the 654 respondents' practices, and $94 \%$ of the respondents having reported either a decrease $(50 \%)$ or unchanged $(44 \%)$ level of use of amalgam, 70\% of the respondent agreed with the statement: 'discontinuation of amalgam restricts a dentist's ability to adequately treat patients'.

Relative to the USA and other countries, in particular Finland, the use of composites in the restoration of posterior teeth was found to be relatively limited, with $93 \%$ of the responding agreeing with the statement: 'providing composites in posterior teeth was more time-consuming than placing a corresponding amalgam restoration', and $81 \%$ of the respondents having considered that: 'the growth in use of composite materials was increasing the total cost of oral health care'. Choice of filling material for posterior teeth was found to be influenced by clinical indications (100\%) and patients 'aesthetic demands' (99\%) preference (95\%) and financial situation (92\%), with a possible reason for the 'low uptake of composite in load-bearing cavities' being considered to be the 'lack of availability to patients treated under Nation Health Services (NHS) regulations.'

Notwithstanding such important considerations, it was found that 'the development of a low-shrink material could result in wider adoption of the use of resin-based tooth-coloured materials for the restoration of posterior teeth, provided that the new resins do not inherit alternative, suboptimal, physical characteristics. As a concluding note it is of interest to note that $92 \%$ of the respondents always, often or sometimes used total etch/dentine bonding, while $45 \%$ of respondents have used a flowable composite 'base'. In contrast, 53\% of the respondents 'never' used rubber dam. As stated in this interesting paper: '..... it may be that dentists who work mainly with amalgam lack experience in using composite.'

Nairn H. F. Wilson, Professor of Restorative Dentistry, King's College London 